Pacific Journal of Mathematics

WALSH SERIES WITH COEFFICIENTS TENDING 


\section{WALSH SERIES WITH COEFFICIENTS TENDING MONOTONICALLY TO ZERO}

\section{J. E. COURY}

Results concerning the Walsh-Fourier coefficients of continuous functions are obtained which extend the work of Bockarev to the case of nonabsolutely convergent Walsh series. Analogues of results for trigonometric series with monotonically decreasing coefficients are proved for the Walsh system. In particular, it is shown that, unlike the trigonometric case, convexity of the coefficients is not sufficient to guarantee that such series are always nonnegative.

1. Introduction. In this paper we study series of the form $S(x)=$ $\sum_{n=0}^{\infty} c_{n} w_{n}(x)$, where $\left\{w_{n}(x)\right\}$ denotes the Walsh system ordered according to Paley's arrangement and $\left\{c_{n}\right\}$ is a sequence of real numbers tending to 0 whose terms satisfy some monotone condition. Bockarev [4] has shown that if the coefficients of $S(x)$ satisfy $\left|c_{0}\right| \geq\left|c_{1}\right| \geq \ldots$ and $S(x)$ is absolutely convergent, then $S(x)$ can be the Walsh-Fourier series of a continuous function only if it is constant. In $\$ 3$ this result is generalized to sequences whose terms eventually satisfy a monotone condition in blocks of length $2^{n}$, and we remove the strong hypothesis that $S(x)$ be absolutely convergent. In particular, it is shown that if $c_{0} \geq c_{1} \geq c_{2} \geq \ldots$ and $2^{n} c_{2^{n}} \rightarrow 0$ as $n \rightarrow \infty$, then $S(x)$ can represent a continuous function $f$ only if $f$ is constant.

In $\$ 4$ we consider the problem of finding a sufficient condition on Walsh series whose coefficients decrease monotonically to 0 which guarantees that the series is everywhere nonnegative. In the case of trigonometric series it is enough to suppose that the sequence $\left\{c_{n}\right\}$ is convex; for the Walsh case, we show that convexity is not sufficient (cf. Yano [13]). We prove, however, that $S(x)$ is nonnegative on [0,1[ if $\left\{c_{n}\right\}$ is assumed to be completely monotone and show that this condition is sufficient that $S(x)$ be Lebesgue integrable.

The last two sections treat Walsh series with completely monotone coefficients in more detail. In $\$ 5$ sufficient conditions are found for $S(x)$ to be a strictly decreasing function on $[0,1[$; it is shown, in particular, that for any fixed $0<t \leq 1 / 2$, the series $\Sigma_{0}^{\infty} t^{n} w_{n}(x)$ is strictly decreasing. In $\S 6$ the differentiability of such series is investigated. Sufficient conditions are given that the series be differentiable almost everywhere, and it is shown that if the derivative exists at a point, then it is necessarily 0 . Thus we prove that the only differentiable Walsh series with completely monotone coefficients are the constants. 
2. Preliminaries. The Rademacher system $\left\{r_{n}(x)\right\}_{n=0}^{\infty}$ on $[0,1[$ is defined as follows: if $x \in\left[0,1\left[\right.\right.$ has the dyadic development $\sum_{n=1}^{\infty} x_{n} 2^{-n}$, where $x_{n}$ is 0 or 1 , then $r_{n}(x)=(-1)^{x_{n+1}}$. The Walsh system, ordered according to Paley's arrangement (see [9]), is obtained by setting $w_{0}(x) \equiv 1$ and defining $w_{n}(x)=r_{n_{1}}(x) \cdot r_{n_{2}}(x) \cdot \ldots \cdot r_{n_{\nu}}(x)$ for $n \geq 1$, where $n=2^{n_{1}}+$ $2^{n_{2}}+\ldots+2^{n_{v}}$ and $n_{1}>n_{2}>\ldots>n_{v} \geq 0$. The functions $\left\{w_{n}(x)\right\}_{n=0}^{\infty}$ form a complete orthonormal set on $\left[0,1\left[\right.\right.$; for $n \geq 1, w_{n}(x)$ is continuous at each dyadic irrational.

Define the Dirichlet kernel for the Walsh system by $D_{k}(x)=$ $\sum_{n=0}^{k-1} w_{n}(x)$. Then $\left|D_{k}(x)\right|<2 / x$ for all $k$ and every $x \in$ ]0, 1[; also, for each $k \geq 1, D_{2 k}(x)=2^{k}$ on $\left[0,2^{-k}\left[\right.\right.$ and $D_{2 k}(x)=0$ on $\left[2^{-k}, 1\right.$ [ (see Fine [5, pp. 386 and 3911).

3. The Walsh-Fourier coefficients of continuous functions. Let $c_{n} \equiv$ $c_{n}(f)$ denote the $(n+1)$ st Walsh-Fourier coefficient of $f(x)$. If $f(x)$ is continuous, then $\left\{c_{n}\right\}$ converges to 0 . Bockarev [4] has shown the following: the only continuous functions on [0,1[ whose Walsh-Fourier coefficients satisfy $\left|c_{0}\right| \geq\left|c_{1}\right| \geq\left|c_{2}\right| \geq \ldots$ with $\Sigma\left|c_{n}\right|<\infty$ are the constants. In this section, we generalize Bockarev's result and show that we need not require the series of coefficients to be absolutely convergent. We begin with a lemma.

LEMMA 1. Let $\left\{c_{n}\right\}$ be a sequence of real numbers and $N$ a fixed but arbitrary positive integer. Then the following inequality holds for each $n \geq N$ and every $x \in\left[2^{-N}, 1[:\right.$

$$
\left|\sum_{k=2^{n}}^{2^{n+1}} c_{k} w_{k}(x)\right| \leq 2^{N+1} \sum_{k=2^{n}}^{2^{n+1}-2}\left|c_{k}-c_{k+1}\right| .
$$

Proof. Using Abel's transformation we obtain

$$
\sum_{k=2^{n}}^{2^{n+1}} c_{k} w_{k}(x)=\sum_{k=2^{n}}^{2^{n+1}-2}\left(c_{k}-c_{k+1}\right) D_{k+1}(x)+c_{2^{n+1}-1} D_{2^{n+1}}(x)-c_{2^{n}} D_{2^{n}}(x)
$$

The functions $D_{2^{n}}(x)$ and $D_{2^{n+1}}(x)$ are 0 on $\left[2^{-N}, 1[\right.$ for every $n \geq N$; also, $\left|D_{m}(x)\right|<2 / x \leq 2^{N+1}$ on $\left[2^{-N}, 1[\right.$ for each $m$. Thus the inequality in the lemma is valid for each $n \geq N$ and every $x \in\left[2^{-N}, 1[\right.$.

THEOREM 1. Let $f(x)$ be continuous on [0, 1[ (except possibly at $x=0$ ), and let $\left\{c_{k}\right\}$ denote the Walsh-Fourier coefficients of $f$. Define 


$$
R_{m}=\sum_{k=2^{m}}^{2^{m+1}-2}\left|c_{k}-c_{k+1}\right|
$$

If

$$
2^{p} \sum_{m=p}^{\infty} R_{m} \rightarrow 0 \text { as } p \rightarrow \infty,
$$

then $f(x)$ is constant.

Proof. Let $S(x)=\Sigma_{k=0}^{\infty} c_{k} w_{k}(x)$. By [5, p. 373], $S_{2^{n}}(x) \rightarrow f(x)$ at each point of continuity of $f$. Define $S_{k}(x+0)$. $=\lim _{\varepsilon \downarrow 0} S_{k}(x+\varepsilon)$ and $S_{k}(x-0)=\lim _{\varepsilon \downarrow 0} S_{k}(x-\varepsilon)$; then $S_{2^{n}}(x+0) \rightarrow f(x)$ and $S_{2^{n}}(x-0) \rightarrow f(x)$ if $x$ is a point of continuity of $f([11, \mathrm{p} .12])$. Since $S_{2^{N}}(x)$ is constant on intervals of the form $\left[i 2^{-N},(i+1) 2^{-N}[\right.$, we have

$$
\begin{aligned}
f\left((i+1) / 2^{N}\right)-f\left(i / 2^{N}\right) & =\sum_{k=N}^{\infty}\left[S_{2^{k+1}}\left((i+1) / 2^{N}-0\right)-S_{2^{k}}\left((i+1) / 2^{N}-0\right)\right] \\
& -\sum_{k=N}^{\infty}\left[S_{2^{k+1}}\left(i / 2^{N}+0\right)-S_{2^{k}}\left(i / 2^{N}+0\right)\right] ;
\end{aligned}
$$

hence

$$
\begin{aligned}
\mid f\left((i+1) / 2^{N}\right) & -f\left(i / 2^{N}\right)\left|\leq \sum_{m=N}^{\infty}\right| \sum_{k=2^{m}}^{2^{m+1}-1} c_{k} w_{k}\left((i+1) / 2^{N}-0\right) \mid \\
& +\sum_{m=N}^{\infty}||_{k=2^{m}}^{2^{m+1}-1} c_{k} w_{k}\left(i / 2^{N}+0\right) \mid .
\end{aligned}
$$

Set $M_{N}=2^{N+1}$; then for $i \geq 1$, Lemma 1 implies that

$$
\left|f\left((i+1) / 2^{N}\right)-f\left(i / 2^{N}\right)\right| \leq 2 \sum_{m=N}^{\infty} M_{N} R_{m}=2 M_{N} \sum_{m=N}^{\infty} R_{m},
$$

and so, for every $i, j \geq 1$ :

$$
\left|f\left(j / 2^{N}\right)-f\left(i / 2^{N}\right)\right|<2^{N+1} M_{N} \sum_{m=N}^{\infty} R_{m} .
$$

Similarly, for any $r \geq N$ and any $i$ for which $i / 2^{r} \geq 1 / 2^{N}$, we have:

$$
\left|f\left((i+1) / 2^{r}\right)-f\left(i / 2^{r}\right)\right| \leq 2 M_{N} \sum_{m=r}^{\infty} R_{m},
$$

and thus for every $i$ and $j$ for which $i / 2^{r}, j / 2^{r} \geq 1 / 2^{N}$ we obtain:

$$
\left|f\left(j / 2^{r}\right)-f\left(i / 2^{r}\right)\right|<2 M_{N} 2^{r} \sum_{m=r}^{\infty} R_{m} .
$$


By hypothesis, the right side of the last inequality tends to 0 as $r \rightarrow \infty$. Thus given any $\varepsilon>0,\left|f\left(j / 2^{r}\right)-f\left(i / 2^{r}\right)\right|<\varepsilon$ for sufficiently large $r$ and all $i, j$ for which $i / 2^{r}, j / 2^{r} \in\left[2^{-N}, 1[\right.$. The set of all such dyadic rationals is dense in $\left[2^{-N}, 1\left[\right.\right.$, and since $f$ is continuous, $|f(x)-f(y)| \leq \varepsilon$ for all $x, y \in\left[2^{-N}, 1[\right.$. Since $\varepsilon$ and $N$ are arbitrary, it follows that $|f(x)-f(y)|=0$ for every $x, y \in] 0,1[$, and so $f$ is constant.

Corollary 1. Let $\left\{c_{n}\right\}$ denote the Walsh-Fourier coefficients of a function $f(x)$ which is continuous on $] 0,1[$. Suppose that $2^{p} \sum_{k=2^{p}}^{\infty}\left|c_{k}-c_{k+1}\right| \rightarrow 0$ as $p \rightarrow \infty$ (in particular, $\left\{c_{n}\right\}$ must be of bounded variation). Then $f(x)$ is constant.

Corollary 2. Suppose that $c_{k} \equiv c_{k}(f)$ satisfies $2^{p} \Sigma_{k=2^{p}}^{\infty}\left|c_{k}\right| \rightarrow 0$ as $p \rightarrow \infty$, and suppose that $f(x)$ is continuous. Then $f(x)$ must be constant.

Proof.

$$
2^{p} \sum_{m=p}^{\infty} R_{m} \leq 2^{p} \sum_{m=p}^{\infty} \sum_{k=2^{m}}^{2^{m+1}-2}\left(\left|c_{k}\right|+\left|c_{k+1}\right|\right) \leq 2^{p+1} \sum_{k=2^{p}}^{\infty}\left|c_{k}\right| \rightarrow 0,
$$

and thus Theorem 1 applies.

We prove next an analogue of Bockarev's result which holds for series whose coefficients are eventually monotone decreasing in blocks of length $2^{n}$. More precisely, we have the following.

THEOREM 2. Suppose that $c_{2^{n}} \geq c_{2^{n+1}} \geq \ldots \geq c_{2^{n+1}-1}$ holds for sufficiently large values of $n$, where $\left\{c_{n}\right\}$ are the Walsh-Fourier coefficients of a continuous function $f(x)$, and suppose that

$$
2^{p} \sum_{m=p}^{\infty}\left(c_{2^{m}}-c_{2^{m+1}-1}\right) \rightarrow 0 \text { as } p \rightarrow \infty .
$$

Then $f(x)$ is constant.

Proof.

$$
2^{p} \sum_{m=p}^{\infty} \sum_{k=2^{m}}^{2^{m+1}}\left|c_{k}-c_{k+1}\right|=2^{p} \sum_{m=p}^{\infty}\left(c_{2^{m}}-c_{2^{m+1}-1}\right) \rightarrow 0
$$

and therefore Theorem 1 applies. 
Corollary 3. (Cf. Bockarev [4]). Suppose $c_{n} \downarrow 0$ and $2^{n} c_{2^{n}} \rightarrow 0$ as $n \rightarrow \infty$ (this holds, for example, if $\left.\Sigma c_{n}<\infty\right)$. Then $f(x)$ continuous on ]0, 1 implies that $f(x)$ is constant.

EXAMPLES. 1. If $c_{n}=1 /(n+1)^{\alpha}$, then $2^{n} c_{2^{n}} \rightarrow 0$ if and only if $\alpha>1$. Thus, $\Sigma_{n=0}^{\infty}(n+1)^{-\alpha} w_{n}(x)$ cannot represent a continuous function for any $\alpha>1$. It is also true that $\Sigma_{n=0}^{\infty}(n+1)^{-1} w_{n}(x)$ is not continuous, but we defer the proof of this fact until $\S 6$.

2. The following shows that Corollary 3 extends Bockarev's result to cases where $\Sigma\left|c_{n}\right|=\infty$. For $2^{n} \leq k<2^{n+1}$ define $c_{k}=1 / n 2^{n}$. It is clear that the hypotheses of Corollary 3 are satisfied, but $\Sigma\left|c_{n}\right|=\Sigma 1 / n=\infty$.

3. The hypotheses of Theorem 1 may be satisfied without satisfying those of Theorem 2: for example, for each $n$ define $c_{2^{n}}=-1 / n 2^{n}$ and let $c_{k}=1 / n 2^{n}$ for $2^{n}<k \leq 2^{n+1}-1$.

4. To show that there exist absolutely convergent series which satisfy the hypothesis of Theorem 2 but not of Bockarev's Theorem, define $c_{k}=0$ for $2^{n} \leq k<2^{n+1}-1$ and $c_{2^{n+1}-1}=-1 /(n-1) 2^{n-1}$. Then $c_{2 n} \geq \ldots \geq c_{2 n+1-1}$ holds for every $n$, and $2^{p} \sum_{m=p}^{\infty}\left(c_{2 m}-c_{2 m+1-1}\right) \rightarrow 0$ since

$$
2^{p} \sum_{m=p}^{\infty}\left|c_{2^{m+1}-1}\right|<\frac{2}{p}+\frac{2}{p-1} \rightarrow 0
$$

However, $\left|c_{0}\right| \geq\left|c_{1}\right| \geq \ldots$ is not satisfied.

4. Sufficient conditions for a nonnegative sum. In the trigonometric case, it is known that if $c_{n} \downarrow 0$ and the sequence $\left\{c_{n}\right\}$ is convex, then $1 / 2 c_{0}+$ $\sum_{k=1}^{\infty} c_{k} \cos k x$ converges (except possibly at $x=0$ ) to a nonnegative and integrable sum $f(x)$ and is the Fourier series of $f(x)$ (see [14, vol. I, p. 183]). Yano [13] has proved a similar result for the Walsh system but fails to get nonnegativity of such series. This is due in part to the fact that the Fejer kernel for the trigonometric system is positive, whereas for the Walsh system it is not (see [5]).

We will show that for a Walsh series $S(x)=\Sigma c_{n} w_{n}(x)$ with $c_{n} \downarrow 0,\left\{c_{n}\right\}$ convex is not sufficient to prove that $S(x)$ is nonnegative; to obtain sufficient conditions for a nonnegative sum, we will work instead with the Abel kernel. We begin with the definition of a completely monotone sequence. 
Definition. (See Lorentz [7, p. 58], Widder [12, p. 108].) Given a sequence $\left\{c_{n}\right\}_{n=0}^{\infty}$ whose terms tend to 0 , define

$$
\Delta^{k} c_{n}=c_{n}-\left(\begin{array}{l}
k \\
1
\end{array}\right) c_{n+1}+\cdots+(-1)^{k} c_{n+k}
$$

for $n, k=0,1, \ldots$. The sequence $\left\{c_{n}\right\}$ is called completely monotone if $\Delta^{k} c_{n} \geq 0$ for all $n, k=0,1, \ldots$. (Thus, for example, $\{1 /(n+1)\}_{n=0}^{\infty}$ and $\left\{t^{n}\right\}_{n=0}^{\infty}$ for $0<t<1$ are completely monotone.)

In particular, if $\left\{c_{n}\right\}$ is completely monotone, then $c_{n} \downarrow 0$ and $\left\{c_{n}\right\}$ is convex.

The moment problem for the space $C[0,1]$ consists in finding a function $g(x)$ of bounded variation for which $\int_{0}^{1} t^{n} d g(t)=c_{n}$ for every $n \geq 0$, where $\left\{c_{n}\right\}$ is given. The proof of the following result may be found in Lorentz [7, p. 59] or Widder [12, p. 108].

THEOREM 3. The moment problem has a nondecreasing and bounded solution $g(x)$ if and only if $\left\{c_{n}\right\}$ is completely monotone.

LEMma 2. Suppose $c_{n} \downarrow 0$. Then $S(x)=\sum_{n=0}^{\infty} c_{n} w_{n}(x)$ converges uniformly on $[\delta, 1$ [ for every $\delta>0 . S(x)$ is continuous except possibly at dyadic rationals.

Proof. Use Abel's transformation, noting that $D(x)=\sum_{k=0}^{\infty} w_{k}(x)$ has uniformly bounded partial sums on every [ $\delta, 1[$ (in fact, $|D(x)| \leq 2 / \delta$ ). Since the Walsh functions are continuous at dyadic irrationals, so therefore is $S(x)$.

LemMa 3. For $0 \leq t<1$, let $P(x, t)=\Sigma_{n=0}^{\infty} t^{n} w_{n}(x)$ denote the Abel kernel and $P_{k}(x, t)=\sum_{n=0}^{k-1} t^{n} w_{n}(x)$ its kth partial sum. Then:

$$
\begin{aligned}
& \text { (i) } P(x, t)=\prod_{n=0}^{\infty}\left(1+t^{2^{n}} r_{n}(x)\right) ; \\
& \text { (ii) } P_{2^{k}}(x, t)=\prod_{n=0}^{k-1}\left(1+t^{2^{n}} r_{n}(x)\right) ; \\
& \text { (iii) } P(x, t)>0 \text { and } P_{2^{k}}(x, t)>0 \text { for } 0 \leq x<1,0 \leq t<1 .
\end{aligned}
$$

Proof. Part (ii) follows by expanding the given product, and hence (i) is true since $P_{2^{k}}(x, t) \rightarrow P(x, t)$. Since each term in the product is positive and since the infinite product cannot diverge to 0 , (iii) follows. 
We prove next the Walsh analogue of Theorem (1.5) in Zygmund [14, p. 183].

THEOREM 4. Let $\left\{c_{n}\right\}$ be a completely monotone sequence. Then the series $\Sigma_{n=0}^{\infty} c_{n} w_{n}(x)$ converges, except possibly at 0 , to a positive and integrable function $S(x)$ and is the Walsh-Fourier series of $S(x)$.

Proof. By Lemma 2, $S(x)$ converges save possibly at 0 . Let $S_{n}$ denote the $n$th partial sum of $S$. Since $\left\{S_{n}\right\}$ is uniformly convergent to $S$ on every $\left[\delta, 1\left[\right.\right.$, we have $S=\lim S_{2^{n}}$. Writing $c_{n}=\int_{0}^{1} t^{n} d g(t)$, we have:

$$
S_{2^{N}}(x)=\sum_{n=0}^{2^{N}-1} c_{n} w_{n}(x)=\int_{0}^{1}\left(\sum_{n=0}^{2^{N}-1} t^{n} w_{n}(x)\right) d g(t)=\int_{0}^{1} P_{2^{N}}(x, t) d g(t)
$$

If $x$ is a dyadic rational, then the factors $1+t^{2^{n}} r_{n}(x)$ in the infinite product $P(x, t)$ are eventually equal to $1+t^{2^{n}}$. Hence $\left\{P_{2^{N}}(x, t)\right\}_{N=0}^{\infty}$ is eventually monotone increasing and so the Monotone Convergence Theorem implies that $S(x)=\int_{0}^{1} P(x, t) d g(t)$. Now for arbitrary $x$ in $] 0,1[$, let $K$ be the least positive integer for which the $K$ th dyadic digit of $x$ is 1 . Then $\left\{P_{2^{N}}(x, t)\right\}$ is bounded by

$$
\prod_{n=0}^{K-1}\left(1+t^{2^{n}} r_{n}(x)\right) \cdot \prod_{n=K}^{\infty}\left(1+t^{2^{n}}\right)
$$

and this infinite product is plainly $P(z, t)$ for a suitable dyadic rational $z \neq 0$. By the previous remark, $S(z)=\int_{0}^{1} P(z, t) d g(t)$ and so $P(z, t)$ is integrable; thus by the Lebesgue Dominated Convergence Theorem we have

$$
S(x)=\lim S_{2^{N}}(x)=\lim \int_{0}^{1} P_{2^{N}}(x, t) d g(t)=\int_{0}^{1} P(x, t) d g(t) .
$$

Appealing to the previous lemma, we conclude that $S(x)>0$ on $[0,1[$.

Since $S_{2^{N}}(x)=\int_{0}^{1} P_{2^{N}}(x, t) d g(t)>0$ for every $N$, Fatou's Lemma gives

$$
\int_{0}^{1}|S(x)| d x=\int_{0}^{1} S(x) d x \leq \lim _{N \rightarrow \infty} \int_{0}^{1} S_{2^{N}}(x) d x=\varliminf_{N \rightarrow \infty} c_{0}=c_{0},
$$

and hence $S(x)$ is integrable.

Let $N$ be fixed but arbitrary; in view of Lemma 2 and the fact that 
$w_{N}(x)=1$ in a neighborhood of 0 , it follows that the partial sums of $\left(1-w_{N}(x)\right) \Sigma_{n=0}^{\infty} c_{n} w_{n}(x)$ converge uniformly to $\left(1-w_{N}(x)\right) S(x)$ on $[0,1[$. Hence

$$
\int_{0}^{1}\left(1-w_{N}(x)\right) S(x) d x=\lim _{n \rightarrow \infty} \int_{0}^{1}\left(1-w_{N}(x)\right) S_{2^{n}}(x) d x=c_{0}-c_{N} .
$$

Integrating the left side gives

$$
\int_{0}^{1} S(x) d x-\int_{0}^{1} S(x) w_{N}(x) d x
$$

where the latter integral is, by definition, the $N$ th Walsh-Fourier coefficient of $S(x)$. Passing to the limit as $N \rightarrow \infty$ and noting that the Walsh-Fourier coefficients of an integrable function converge to 0 ([11, p. 14]), we conclude that $\int_{0}^{1} S(x) d x=c_{0}$. Thus we also have $c_{N}=\int_{0}^{1} S(x) w_{N}(x) d x$ for every value of $N$, and hence $\Sigma c_{n} w_{n}(x)$ is the Walsh-Fourier series of $S(x)$.

REMARK. It is not necessary that $\left\{c_{n}\right\}$ be completely monotone in order for $S(x)$ to be positive on $\left[0,1\left[\right.\right.$. To show this, let $\left\{c_{n}\right\}$ be any sequence for which $c_{n} \downarrow 0$ and $c_{k} \geq \sum_{n=k+1}^{\infty} c_{n}$ for every $k \geq 0$. Plainly $S(x)$ is positive, but $\left\{c_{n}\right\}$ need not be completely monotone. Take, for example, $c_{0}=4 / 3, c_{1}=5 / 6$, and $c_{n}=1 / 2^{n}$ for $n \geq 2$; then $\left\{c_{n}\right\}$ is not even convex.

To show, however, that there exist convex sequences for which $S(x)$ is not positive requires a more delicate argument.

Proposition 1. There exist convex sequences $\left\{c_{n}\right\}$ for which $S(x)=\sum_{n=0}^{\infty} c_{n} w_{n}(x)$ assumes negative values.

Proof. Define $d g(t)=-1$ for $0 \leq t<1 / 2$ and $d g(t)=1$ for $1 / 2 \leq t$ $<1$. Set $a_{n}=\int_{0}^{1} t^{n} d g(t)=\left(1-1 / 2^{n}\right) \div(n+1)$; it is easily checked that $\left\{a_{n}\right\}_{n=2}^{\infty}$ is convex. Let $c_{n}=a_{n+2}$ for $n \geq 0$. We will show that $S(1-0)=$ $\lim _{\varepsilon \downarrow 0} S(1-\varepsilon)$ is negative. Since $S(1-0)=\int_{0}^{1} t^{2} P(1-0, t) d g(t)$, where $P(1-0, t)=\Pi_{n=0}^{\infty}\left(1-t^{2^{n}}\right)$, it suffices to show that

$$
\int_{0}^{1 / 2} t^{2} P(1-0, t) d t>\int_{1 / 2}^{1} t^{2} P(1-0, t) d t,
$$

or equivalently, adding $\int_{0}^{1 / 2} t^{2} P(1-0, t) d t$ to each side of this inequality, that

$$
2 \int_{0}^{1 / 2} t^{2} P(1-0, t) d t>\int_{0}^{1} t^{2} P(1-0, t) d t .
$$

For $t \in[0,1 / 2]$ we have 


$$
\begin{aligned}
P(1-0, t)=1-t-t^{2}+t^{3} \ldots & \geq 1-t-t^{2}+t^{3}-\left(t^{4}+t^{5}+\ldots\right) \\
& \geq 1-t-t^{2}
\end{aligned}
$$

thus

$$
\begin{aligned}
P(1-0, t) & =\prod_{n=0}^{\infty}\left(1-t^{2^{n}}\right)=(1-t) \prod_{n=1}^{\infty}\left(1-t^{2^{n}}\right)=(1-t) P\left(1-0, t^{2}\right) \\
& \geq(1-t)\left(1-t^{2}-t^{4}\right)=1-t-t^{2}+t^{3}-t^{4}+t^{5}
\end{aligned}
$$

hence

$$
\begin{gathered}
2 \int_{0}^{1 / 2} t^{2} P(1-0, t) d t \geq 2 \int_{0}^{1 / 2}\left(t^{2}-t^{3}-t^{4}+t^{5}-t^{6}+t^{7}\right) d t \\
\simeq .043536 \ldots>.0435 .
\end{gathered}
$$

Also,

$$
\begin{aligned}
P(1-0, t) & \leq(1-t)\left(1-t^{2}\right)\left(1-t^{4}\right) \\
& =1-t-t^{2}+t^{3}-t^{4}+t^{5}+t^{6}-t^{7}
\end{aligned}
$$

hence

$$
\begin{aligned}
\int_{0}^{1} t^{2} P(1-0, t) d t & \leq \frac{1}{3}-\frac{1}{4}-\frac{1}{5}+\frac{1}{6}-\frac{1}{7}+\frac{1}{8}+\frac{1}{9}-\frac{1}{10} \\
& \simeq .043254 . \ldots<.0433 .
\end{aligned}
$$

(The author wishes to thank Professor D. Boyd for the above estimates on $P(1-0, t)$.)

We close this section with the Walsh analogues of two results that hold for trigonometric series whose coefficients tend monotonically to 0 .

Proposition 2. There exist Walsh-Fourier series with coefficients decreasing monotonically to 0 arbitrarily slowly.

Proof. In view of Yano's result $\left[13\right.$, p. 235] that $\left\{c_{n}\right\}$ convex guar- 
antees the integrability of $S(x)$, it is enough to show that given an arbitrary sequence of positive numbers $\varepsilon_{n} \rightarrow 0$, there exists a convex sequence $\left\{c_{n}\right\}$ with $c_{n} \geq \varepsilon_{n}$ and $c_{n} \rightarrow 0$. Such a series can easily be constructed geometrically (see, for example, Bary [3, vol. II, p. 203]).

Proposition 3. (Cf. Zygmund [14, vol. I, p. 184].) There exists a Walsh series $S(x)=\Sigma c_{n} w_{n}(x)$ with $c_{n} \downarrow 0$ such that $S(x)$ is not Lebesgue integrable.

The proof of this result is essentially the same as in the trigonometric case: it relies on the fact that the Lebesgue constants $L_{k}=\int_{0}^{1}\left|D_{k}(x)\right| d x$ for the Walsh system satisfy $L_{k}=O(\log k)([5, \mathrm{p} .391])$.

The proof of Proposition 3 may also be modified to show that convexity of $\left\{c_{n}\right\}$ is not necessary for the integrability of $S(x)$ : for any fixed $x \neq 0$, summation by parts gives

$$
S(x)=\sum_{k=0}^{\infty}\left(c_{k}-c_{k+1}\right) D_{k+1}(x),
$$

where $D_{k}(x)$ denotes the $k$ th partial sum of the Dirichlet kernel. If the sequence $\left\{c_{n}\right\}$ is chosen so that $c_{k} \downarrow 0$ and is constant for $2^{n} \leq k<2^{n+1}$ $(n \geq 0)$, we obtain

$$
S(x)=\sum_{k=0}^{\infty}\left(c_{2^{k}-1}-c_{2^{k}}\right) D_{2^{k}}(x) .
$$

Since $D_{2^{k}}(x)$ equals $2^{k}$ on $\left[0,2^{-k}\left[\right.\right.$ and is 0 on $\left[2^{-k}, 1\right.$, follows that $S(x)$ is nonnegative. Hence, by the Monotone Convergence Theorem:

$$
\begin{aligned}
\int_{0}^{1}|S(x)| d x=\int_{0}^{1} S(x) d x & =\sum_{k=0}^{\infty}\left(c_{2^{k}-1}-c_{2^{k}}\right) \int_{0}^{1} D_{2^{k}}(x) d x \\
& =\sum_{k=0}^{\infty}\left(c_{2^{k}-1}-c_{2^{k}}\right)=c_{0} .
\end{aligned}
$$

Thus $S(x)$ is integrable, but the sequence $\left\{c_{n}\right\}$ is plainly not convex.

5. Strictly decreasing series. In this section we will find sufficient conditions on the coefficients of $S(x)$ to ensure that $S(x)$ is a strictly decreasing function on [0, 1[. We begin with the following result. (Cf. Balasov [1].) 
THEOREM 5. Let $S(x)=\Sigma c_{n} w_{n}(x)$ with $c_{n} \downarrow 0$, and suppose that $c_{n} \geq \sum_{k=n+1}^{\infty} c_{k}$ for every $n \geq 0$. Then $S(x)$ is strictly decreasing on $[0,1[$.

Proof. Suppose that $x<y$ and that the first dyadic digit in which $x$ and $y$ differ is the $(M+1)$ st. Since $x$ and $y$ coincide in the first $M$ positions, $w_{n}(x)=w_{n}(y)$ for $n=0,1, \ldots, 2^{M}-1$. Also, $x<y$ implies that $x_{M+1}=0$ and $y_{M+1}=1$, and hence $w_{2 M}(x)=1, w_{2 M}(y)=-1$. Thus the hypothesis that $c_{n} \geq \sum_{k=n+1}^{\infty} c_{k}$ for each $n$ guarantees that $S(x)$ is at least monotone nonincreasing. To show that it is strictly decreasing, it is enough to prove the sequence $\left\{w_{n}(x)\right\}_{n=0}^{\infty}$ can never be eventually equal to 1 unless $x=0$.

We will show, in fact, that if two sequences $\left\{w_{n}(x)\right\}_{0}^{\infty}$ and $\left\{w_{n}(y)\right\}_{0}^{\infty}$ coincide in all but finitely many terms, then $x=y$. Let $N$ be such that $w_{n}(x)$ $=w_{n}(y)$ for every $n \geq N$. For $m \geq N-1$, we have $w_{2^{m}}(x)=w_{2^{m}}(y)$ since $2^{m} \geq N$; hence $r_{m}(x)=r_{m}(y)$ and thus $x_{m+1}=y_{m+1}$ for every $m \geq N-1$. For a fixed but arbitrary $m \geq N-1$, set $k=2^{m}+2^{j}$, where $0 \leq j \leq N-2$. Then $w_{k}(x)=r_{m}(x) \cdot r_{j}(x)$ and $w_{k}(y)=r_{m}(y) \cdot r_{j}(y)$, and hence $r_{j}(x)=r_{j}(y)$, from which we conclude that $x_{j+1}=y_{j+1}$. Since $j \in\{0,1, \ldots, N-2\}$ was arbitrary, it follows that $x_{n}=y_{n}$ for every $n$ and hence that $x=y$.

COROLlaRY 4. Suppose that $\left\{c_{n}\right\}$ is a completely monotone sequence whose associated measure is supported on $[0,1 / 2]$. Then $S(x)=\Sigma c_{n} w_{n}(x)$ is strictly decreasing on $[0,1[$.

Proof. For each $n \geq 0$,

$$
c_{n}-\sum_{k=n+1}^{\infty} c_{k}=\int_{0}^{1}\left(t^{n}-\sum_{k=n+1}^{\infty} t^{k}\right) d g(t)=\int_{0}^{1} t^{n}\left(1-\frac{t}{1-t}\right) d g(t) .
$$

Since the integrand in the last expression is nonnegative for $0 \leq t \leq 1 / 2$, the conclusion follows from Theorem 5 .

Since the sequence $\left\{a^{n}\right\}_{n=0}^{\infty}$ is completely monotone, $d g(t)$ being the point mass concentrated at $a$, we have the following result.

Corollary 5. Let $0<a \leq 1 / 2$ be fixed. Then the series $\sum_{n=0}^{\infty} a^{n} w_{n}(x)$ is strictly decreasing on $[0,1[$.

6. Differentiable sums. Let $S(x)$ be a Walsh series whose coefficients form a completely monotone sequence. In this section we give sufficient 
conditions for $S(x)$ to possess a derivative at almost all points of [0,1[, and we show that the only such series that are differentiable are the constants. We begin with a definition.

Definition. Let $\mu$ be a measure on [0, 1[ with support $P$. Define the essential supremum of the support of $\mu$ by

$$
\Omega(P)=\inf \{\lambda: \mu(P \cap[\lambda, 1])=0\}
$$

The next proposition shows that if the support of the measure for a completely monotone sequence is bounded away from 1 , then the series cannot be continuous.

Proposition 4. Let $\left\{c_{n}\right\}$ be a completely monotone sequence whose corresponding measure is supported on a set $E$, and suppose that $\Omega(E)<1$. Then $\Sigma c_{n}$ is convergent and hence $S(x)=\Sigma c_{n} w_{n}(x)$ cannot be continuous unless it is constant.

Proof. By the Monotone Convergence Theorem,

$$
\begin{aligned}
\sum_{n=0}^{\infty} c_{n} & =\sum_{n=0}^{\infty} \int_{E} t^{n} d g(t)=\int_{E}\left(\sum_{n=0}^{\infty} t^{n}\right) d g(t)=\int_{E} \frac{1}{1-t} d g(t) \\
& \leq \int_{0}^{\Omega(E)} \frac{1}{1-t} d g(t) \leq \frac{1}{1-\Omega(E)} \int_{0}^{\Omega(E)} d g(t)=\frac{1}{1-\Omega(E)} c_{0}<\infty .
\end{aligned}
$$

That $S(x)$ is not continuous now follows from Corollary 3 .

Corollary 6. Let $\left\{c_{n}\right\}$ be completely monotone with $\Omega(E)<1$, and suppose that $S(x)=\Sigma c_{n} w_{n}(x)$ is not constant. Then $S(x)$ is not differentiable.

In the case where $\Omega(E)=1$, it may still happen that $S(x)$ is discontinuous and hence nondifferentiable, as shown by the next result.

Proposition 5. The series $S(x)=\sum_{n=0}^{\infty}(n+1)^{-1} w_{n}(x)$ is not continuous.

Proof. We show that $S(1 / 2-0)>S(1 / 2+0)$. Since $\{1 /(n+1)\}_{0}^{\infty}$ corresponds to the distribution function $g(t)=t$, we have: 


$$
S(1 / 2+0)=\int_{0}^{1} P(1 / 2+0, t) d t=\int_{0}^{1}(1-t) \prod_{n=1}^{\infty}\left(1+t^{2^{n}}\right) d t=\ln 2 \simeq .69
$$

We may estimate $S(1 / 2-0)$ as follows:

$$
\begin{gathered}
S(1 / 2-0)=\int_{0}^{1} P(1 / 2-0, t) d t=\int_{0}^{1}(1+t)\left(1-t^{2}\right)\left(1-t^{4}\right) \ldots d t \\
>\int_{0}^{3 / 4}\left(1+t-t^{2}-t^{3} \ldots\right) d t \simeq .81 .
\end{gathered}
$$

Thus $S(x)$ is discontinuous at $x=1 / 2$.

For a suitable restriction, however, on $\Omega(E)$ or on the coefficients of $S(x)$, we do get differentiability of $S(x)$ almost everywhere.

THEOREM 6. Suppose that $\left\{c_{n}\right\}$ satisfies one of the following:

(i) $c_{n} \geq \sum_{k=n+1}^{\infty} c_{k}$ for every $n \geq 0$;

(ii) $\left\{c_{n}\right\}$ is completely monotone with $\Omega(E)<1$.

Then $S(x)=\Sigma c_{n} w_{n}(x)$ is differentiable at almost all points of [0, 1[. Further, if (ii) holds, then $S^{\prime}(x)=0$ a.e.

Proof. If (i) holds, Theorem 5 implies that $S(x)$ is monotone and hence has a derivative almost everywhere.

Suppose now that (ii) is satisfied. By the strong law of large numbers, $\lim _{k \rightarrow \infty}\left(x_{1}+x_{2}+\ldots+x_{k}\right) / k=1 / 2$ for almost all $x=\sum_{k=1}^{\infty} x_{k} 2^{-k}$ in [0, 1[. Denote by $T$ the set of all points for which this relation holds, and let $x$ belong to $T$. Then for all sufficiently large values of $k$, there exists an integer $i$ such that $k / 3 \leq i \leq k$ and $x_{i}=0$ : for otherwise,

$$
\frac{x_{1}+\cdots+x_{k}}{k} \geq \frac{k-([k / 3]+1)}{k} \rightarrow 2 / 3 \text { as } k \rightarrow \infty \text {, }
$$

contrary to our assumption that $x \in T$.

Let $x \in T$ be fixed; for $h>0$, denote by $N(x, h)$ the largest integer such that $x+h$ and $x$ coincide in the first $N(x, h)$ coordinates. Let $M(h)$ denote the first coordinate of $h$ in which a 1 appears. If $x_{n}=0$ for some $n \leq$ $M(h)$, then evidently $N(x, h) \geq n--1$. In view of the preceding paragraph, if $h$ is sufficiently small (so that $M(h)$ is sufficiently large), we can find an integer $i$ such that $M(h) / 3 \leq i \leq M(h)$ and $x_{i}=0$. Thus for fixed but arbitrary $x \in T$, we have $N(x, h) \geq M(h) / 3$. (This is hardly a precise estimate of $N(x, h)$, but it is sufficient for our purposes.)

We now show that $S^{\prime}(x)$ exists and equals 0 for every $x \in T$. To 
simplify notation, we will write $N$ for $N(x, h)$. Then

$$
\begin{gathered}
\frac{S(x+h)-S(x)}{h}=\frac{1}{h} \int_{0}^{1}[P(x+h, t)-P(x, t)] d g(t) \\
=\frac{1}{h} \int_{0}^{1} \prod_{0}^{N-1}\left(1+t^{2^{n}} r_{n}(x)\right)\left\{\prod_{N}^{\infty}\left(1+t^{2^{n}} r_{n}(x+h)\right)-\prod_{N}^{\infty}\left(1+t^{2^{n}} r_{n}(x)\right)\right\} d g(t) .
\end{gathered}
$$

The expression in braces may be estimated by

$$
\begin{aligned}
\prod_{N}^{\infty}\left(1+t^{2^{n}} r_{n}(x+h)\right)- & \prod_{N}^{\infty}\left(1+t^{2^{n}} r_{n}(x)\right) \leq \prod_{N}^{\infty}\left(1+t^{2^{n}}\right)-\prod_{N}^{\infty}\left(1-t^{2^{n}}\right) \\
& \leq 2\left[t^{2^{N}}+t^{2 \cdot 2^{N}}+t^{3 \cdot 2^{N}}+\cdots\right] \\
& =2 \frac{t^{2^{N}}}{1-t^{2^{N}}}
\end{aligned}
$$

Define $C=P(0, \Omega(E))=\Pi_{n=0}^{\infty}\left(1+\Omega(E)^{2^{n}}\right)$; assuming $\Omega(E)<1$, we then have:

$$
\begin{gathered}
\left|\frac{S(x+h)-S(x)}{h}\right| \leq \frac{2}{h} \int_{0}^{\Omega(E)} \prod_{n=0}^{N-1}\left(1+t^{2^{n}} r_{n}(x)\right) \frac{t^{2^{N}}}{1-t^{2^{N}}} d g(t) \\
\leq \frac{2}{h} \cdot \frac{\Omega(E)^{2^{N}}}{1-\Omega(E)^{2^{N}}} \int_{0}^{\Omega(E)} \prod_{n=0}^{N-1}\left(1+t^{2^{n}} r_{n}(x)\right) d g(t) \\
\leq \frac{2}{h} \cdot \frac{\Omega(E)^{2^{N}}}{1-\Omega(E)^{2^{N}}} C \int_{0}^{\Omega(E)} d g(t) \\
\quad=\frac{2 \cdot C}{h} \cdot \frac{\Omega(E)^{2^{N}}}{1-\Omega(E)^{2^{N}}-c_{0} .}
\end{gathered}
$$

We show that this last expression tends to 0 as $h \downarrow 0$. By the previous paragraph, $N \equiv N(x, h) \geq M(h) / 3$. Since $M(h)$ is asymptotically equal to $\log _{2} 1 / h$ as $h \downarrow 0$, it follows that $\Omega(E)^{2^{N}} / h \rightarrow 0$ as $h \downarrow 0$. Also, since $N(x, h) \rightarrow$ $\infty$ as $h \downarrow 0$, we have $1 /\left(1-\Omega(E)^{2^{N}}\right) \rightarrow 1$ as $h \downarrow 0$. Thus we conclude $S_{+}^{\prime}(x)$ is 0 at each point of $T$. 
Similarily, we show $S_{-}^{\prime}(x)$ is 0 on the set $-T$. Hence $S^{\prime}(x)$ exists and is 0 at every point of $T \cap(-T)$, that is, at every point of $T$.

REMarKs. 1. It should be noted that the set $T$ in the preceding proof contains no dyadic rationals, and neither does it contain every dyadic irrational ( $T$ is in fact a set of the first category; see [8, p. 85]). However, as shown in the next result, if $\Omega(E)<1$ and the derivative of $S(x)$ exists at any point, then it is necessarily 0 at that point.

2. While the preceding proof does not give differentiability almost everywhere for the case in which $\Omega(E)=1$, a similar approach can be used to show that, except possibly at dyadic rationals, the derivative of $S(x)$ is 0 whenever it exists.

LEMMA 4. Let $\left\{c_{n}\right\}$ be completely monotone.

(i) Suppose $\Omega(E)<1$. Then $S^{\prime}(x)=0$ whenever the derivative exists.

(ii) Suppose $\Omega(E)=1$. If $S^{\prime}(x)$ exists, then $S^{\prime}(x) \leq 0$. If, in addition, $x$ is a dyadic irrational, then $S^{\prime}(x)=0$.

Proof. If $S^{\prime}\left(x_{0}\right)$ exists, it may be expressed as

$$
\begin{gathered}
S^{\prime}\left(x_{0}\right)=\lim _{N \rightarrow \infty} 2^{N} \int_{0}^{1}\left[P\left(x_{0}+\frac{1}{2^{N}}, t\right)-P\left(x_{0}, t\right)\right] d g(t) \\
=\lim _{N \rightarrow \infty} 2^{N} \int_{0}^{1} 2 \varepsilon_{N} t^{t^{N-1}} \prod_{\substack{n=0 \\
n \neq N-1}}^{\infty}\left(1+t^{2^{n}} r_{n}\left(x_{0}\right)\right) d g(t),
\end{gathered}
$$

where $\varepsilon_{N}$ is +1 or -1 according as $x_{0}$ has 1 or 0 in its $N$ th coordinate.

If $x_{0}$ is a dyadic irrational, there are infinitely many 0 's and 1's in its dyadic expansion; thus, since the limit is assumed to exist, it must equal 0 . If $x_{0}$ is a dyadic rational, then $\varepsilon_{N}$ is eventually -1 , and hence $S^{\prime}\left(x_{0}\right) \leq 0$.

Now suppose that $\Omega(E)<1$. From the above expression for $S^{\prime}\left(x_{0}\right)$, it follows that

$$
\begin{gathered}
\left|S^{\prime}\left(x_{0}\right)\right| \leq \lim _{N \rightarrow \infty} 2^{N+1} \Omega(E)^{2^{N-1}} \prod_{\substack{n=0 \\
n \neq N-1}}^{\infty}\left(1+\Omega(E)^{2^{n}}\right) \int_{0}^{1} d g(t) \\
\leq c_{0} \prod_{n=0}^{\infty}\left(1+\Omega(E)^{2^{n}}\right) \lim _{N \rightarrow \infty} 2^{N+1} \Omega(E)^{2^{N-1}}=0,
\end{gathered}
$$

since $\Omega(E)<1$. 
COROLlary 7. The only absolutely continuous functions whose Walsh-Fourier series have completely monotone coefficients are the constants.

THEOREM 7. Let $S(x)$ be a Walsh series with completely monotone coefficients, and suppose that $S(x)$ is differentiable on $] 0,1[$. Then $S(x)$ is constant on $] 0,1[$.

Proof. Suppose $S(x)$ is differentiable on ]0, 1[. Then $S(x)$ is continuous on ]0, $1\left[\right.$ and also, by Lemma $4, S^{\prime}(x)=0$ at every dyadic irrational. Since $S^{\prime}(x)$ is integrable, it follows that $S(x)$ is absolutely continuous, and therefore constant, on every closed subinterval of ]0, $1[$ (see [6, (18.41)]). Hence $S(x)$ is constant on ]0, 1 .

\section{REFERENCES}

1. L. A. Balasov, Series with gaps (Russian), Izv. Akad. Nauk SSSR Ser. Mat., 29 (1965), 631-644.

2. __ On series with respect to a Walsh system with monotone coefficients, Siberian Math. J., 12 (1971), 18-28.

3. N. K. Bary, A Treatise on Trigonometric Series (2 volumes), Macmillan, New York, 1964.

4. S. V. Bockarev, On the Fourier-Walsh coefficients, Math. USSR-Izvestija, 4 (1970), 209-214.

5. N. J. Fine, On the Walsh functions, Trans. Amer. Math. Soc., 65 (1949), 372-414.

6. E. Hewitt and K. Stromberg, Real and Abstract Analysis, Springer-Verlag, Berlin, 1965.

7. G. G. Lorentz, Bernstein Polynomials, University of Toronto Press, Toronto, 1953.

8. J. C. Oxtoby, Measure and Category, Springer-Verlag, Berlin, 1971.

9. R. E. A. C. Paley, A remarkable system of orthogonal functions, Proc. London Math. Soc., 34 (1932), 241-279.

10. A. A. Sneider, On series with respect to Walsh functions with monotone coefficients (Russian), Izv. Akad. Nauk SSSR Ser. Mat., 12 (1948), 179-192.

11. J. L. Walsh, A closed set of normal orthogonal functions, Amer. J. Math., 55(1923), 5-24.

12. D. V. Widder, The Laplace Transform, Princeton Univ. Press, Princeton, New Jersey, 1941.

13. S. Yano, On Walsh-Fourier series, Tohoku Math. J., 3 (1951), 223-242.

14. A. Zygmund, Trigonometric Series, 2nd Edition (2 volumes), Cambridge University Press, Cambridge, England, 1968.

Received June 8, 1973. The author expresses his thanks to the referee for his suggestions and comments. This research supported by NRC Grant A8131.

UNIVERSITY OF BRITISH COLUMBIA 


\section{PACIFIC JOURNAL OF MATHEMATICS}

\section{EDITORS}

RICHARD ARENS (Managing Editor)

University of California

Los Angeles, California, 90024

\section{R. A. BEAUMONT}

University of Washington

Seattle, Washington 98105

\section{J. DugunDJI}

Department of Mathematics University of Southern California Los Angeles, California 90007

D. Gillbarg AND J. MilgRAM

Stanford University Stanford, California 94305

\section{ASSOCIATE EDITORS}

E. F. BECKENBACH

B. H. NEUMANN

SUPPORTING

UNIVERSITY OF BRITISH COLUMBIA

UNIVERSITY OF CALIFORNIA

MONTANA STATE UNIVERSITY

UNIVERSITY OF NEVADA

NEW MEXICO STATE UNIVERSITY

OREGON STATE UNIVERSITY

UNIVERSITY OF OREGON OSAKA UNIVERSITY
F. WOLF

K. YosHIDA

INSTITUTIONS

UNIVERSITY OF SOUTHERN CALIFORNIA

STANFORD UNIVERSITY

UNIVERSITY OF TOKYO

UNIVERSITY OF UTAH

WASHINGTON STATE UNIVERSITY

UNIVERSITY OF WASHINGTON

AMERICAN MATHEMATICAL SOCIETY

NAVAL WEAPONS CENTER

The Supporting Institutions listed above contribute to the cost of publication of this Journal, but they are not owners or publishers and have no responsibility for its content or policies.

Mathematical papers intended for publication in the Pacific Journal of Mathematics should be in typed form or offset-reproduced, (not dittoed), double spaced with large margins. Underline Greek letters in red, German in green, and script in blue. The first paragraph or two must be capable of being used separately as a synopsis of the entire paper. Items of the bibliography should not be cited there unless absolutely necessary, in which case they must be identified by author and Journal, rather than by item number. Manuscripts, in duplicate if possible, may be sent to any one of the five editors. Please classify according to the scheme of Math. Rev. Index to Vol. ${ }^{39}$. All other communications to the editors should be addressed to the managing editor, or Elaine Barth, University of California, Los Angeles, California, 90024.

100 reprints are provided free for each article, only if page charges have been substantially paid. Additional copies may be obtained at cost in multiples of 50 .

The Pacific Journal of Mathematics is issued monthly as of January 1966. Regular subscription rate: $\$ 72.00$ a year (6 Vols., 12 issues). Special rate: $\$ 36.00$ a year to individual members of supporting institutions.

Subscriptions, orders for back numbers, and changes of address should be sent to Pacific Journal of Mathematics, 103 Highland Boulevard, Berkeley, California 90708.

\section{PUBLISHED BY PACIFIC JOURNAL OF MATHEMATICS, A NON-PROFIT CORPORATION}

Copyright (C) 1974 by Pacific Journal of Mathematics

Manufactured and first issued in the U.S.A. 


\section{Pacific Journal of Mathematics}

\section{Vol. 54, No. $2 \quad$ June, 1974}

John Edward Coury, Walsh series with coefficients tending monotonically to

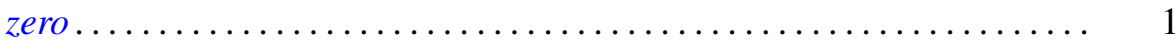

Patrick Michael Fitzpatrick and Walter Volodymyr Petryshyn, Fixed point theorems for multivalued noncompact acyclic mappings ............

Irving Leonard Glicksberg, More on Phragmén-Lindelöf for function

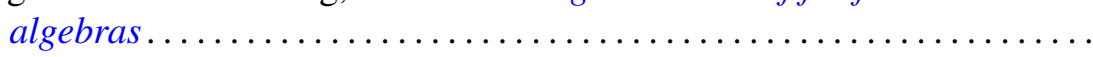

Adilson Goncalves, Structural constants. II .................. 39

Richard P. Gosselin, Closure theorems for affine transformation groups .... 53

Ralph Peter Grimaldi, Baer and UT-modules over domains ........... 59

Edward Grossman, On the prime ideal divisors of $\left(a^{n}-b^{n}\right) \ldots \ldots \ldots \ldots . \ldots 73$

A. Hedayat and Ester Seiden, On the theory and application of sum composition of Latin squares and orthogonal Latin squares.......... .

Gerald L. Itzkowitz, Continuous measures, Baire category, and uniform continuity in topological groups ......................... 115

Francis Masat, Right simple congruences on a semigroup ............ 127

Robert Harvey Oehmke, Right congruences and semisimplicity for Rees matrix semigroups..................................

Qazi Ibadur Rahman and Jan Stankiewicz, Differential inequalities and local valency . . . . . . . . . . . . . . . . . . . . . . . . . . . . . . . . . . . . . 165

William John Reed, Random points in a simplex ................ 183

Mohan S. Shrikhande, Strongly regular graphs and group divisible

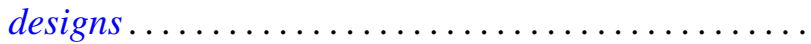

Zahava Shmuely, The structure of Galois connections ... . .

Robert C. Shock, Dual generalizations of the Artinian and Noetherian

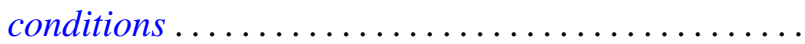

Arne Stray, Approximation and interpolation for some spaces of analytic functions in the unit disc ........................

Eldon Jon Vought, Monotone decompositions into trees of Hausdorff continua irreducible about a finite subset ............

James Wirth, The mapping cylinder axiom for WCHP fibrations ... 263

Gordon S. Woodward, Invariant means and ergodic sets in Fourier analysis... 